\title{
Advance Analytical Approach to Characterize Charge Variants in Monoclonal Antibodies
}

\author{
Bhatt Mithun ${ }^{1 *}$, Mehta Kavit ${ }^{2}$, Bhatt Keyur ${ }^{3}$ and Shekhawat Rakesh ${ }^{4}$ \\ ${ }^{1}$ PhD Scholar, Faculty of Science, Ganpat University, Mehsana, Gujarat, India \\ ${ }^{2}$ Research Guide and Assistant Professor, Faculty of Science, Ganpat University, Mehsana, Gujarat, India \\ ${ }^{3}$ Research Coordinator and Assistant Professor, Faculty of Science, Ganpat University, Mehsana, Gujarat, India \\ ${ }^{4}$ Senior General Manager, R\&D Department, Intas Biopharmaceuticals, Ahmedabad, Gujarat, India
}

\begin{abstract}
Post-translational modifications induce variations of monoclonal antibodies charge distribution that can potentially affect their biological activity. The characterization and the monitoring of these charge variants are critical quality requirements to ensure stability, process consistency and product quality. Charge variants are usually characterized by preparative ion exchange chromatography, collection of fractions and subsequent reverse-phase liquid chromatography with mass spectrometry analysis which is time consuming. A method combining highly selective cation exchange chromatographybased charge variant analysis with on-line mass spectrometric (MS) detection reduce complex and time consuming characterization methods. For this reason, a straightforward on-line charge variant analysis method is highly desirable and analytical laboratories are actively pursuing efforts to overcome this challenge. The objective of study is to develop analytical method of Charge Variant Analysis-MS (CVA-MS) method which will be based on volatile chromatographic buffers with relatively low ionic strength that enable on-line TOF mass spectrometric detection of charge variants of multiple mAbs. A feature of the method would be the use of low ionic strength eluents, which are a prerequisite for successful MS detection, but which exhibit relatively low buffering capacity. From a single injection without any sample preparation, substantial amounts of data can be obtained, which would normally require multiple modes of analysis with several sample preparation techniques. Thus, Charge Variant Analysis-Mass Spectrometry technique will allows the monitoring of multiple attributes on the intact protein level. Having applied Charge Variant Analysis-Mass Spectrometry for the analysis of different mAbs, we can find out novel information on antibody heterogeneity and on its susceptibility to degradation, which can have fundamental implications for the introduction of potential biosimilar candidates in the future. In this study, a mixed mode ion exchange chromatographic method using volatile salts and coupled on-line to native mass spectrometry will be developed in association with a middle-up approach for a detailed characterization of monoclonal antibodies charge variants.
\end{abstract}

\section{Keywords}

Monoclonal antibodies, Charge variant analysis, Cation exchange chromatography, High resolution mass spectrometry

\section{Introduction}

\section{Origin/background}

Proteins are made up of chains containing numerous amino acids, several of which possess acidic or basic side chain functionalities. This results in an overall charge on the surface of the protein that can be controlled by adjusting the $\mathrm{pH}$ of the surrounding solution. The isoelectric point, $\mathrm{pl}$, is the $\mathrm{pH}$ at which the net charge of the protein is neutral (the number of positive charges is equal to the number of negative charges). If the $\mathrm{pH}$ is below this value, the protein will possess an overall positive charge and can be retained on a negatively charged cation-exchange sorbent; if the $\mathrm{pH}$ is above the $\mathrm{pl}$, the protein will be negatively charged overall and can be retained on an anion-exchange sorbent.

Quality by design ( $Q b D$ ) is a new approach to develop and to manufacture biopharmaceutical products. QbD guarantees product quality and ensures that a consistent product with

*Corresponding author: Bhatt Mithun, PhD Scholar, Faculty of Science, Ganpat University, Mehsana, Gujarat, India

Accepted: February 20, 2021

Published online: February 22, 2021

Citation: Mithun B, Kavit M, Keyur B, et al. (2021) Advance Analytical Approach to Characterize Charge Variants in Monoclonal Antibodies. J Ind Biotechnol 2(1):7-9 
preferred quality attributes is generated [1].

MAbs and related products are subject to a wide variety of modifications including glycosylation, lysine truncation, oxidation, deamidation, disulfide bond scrambling, and fragmentation, aggregation, glycation, isomerization and pyroglutamate formation. Some modifications can affect drug efficacy and safety, and can thus be considered critical quality attributes (CQAs). CQAs are directly related to the components present in and the physiochemical conditions of the cell culture media at the time of fermentation and can be influenced by critical process parameters [2-4].

\section{Objective}

The objective of study is to develop analytical method of Charge Variant Aanlysis-MS method which will be based on volatile chromatographic buffers with relatively low ionic strength that enable on-line TOF mass spectrometric detection of charge variants of multiple mAbs. A feature of the method would be the use of low ionic strength eluents, which are a prerequisite for successful MS detection, but which exhibit relatively low buffering capacity. From a single injection without any sample preparation, substantial amounts of data can be obtained, which would normally require multiple modes of analysis with several sample preparation techniques. Thus, Charge Variant Analysis-Mass Spectrometry technique will allows the monitoring of multiple attributes on the intact protein level. Having applied Charge Variant Analysis-Mass Spectrometry for the analysis of different mAbs, we can find out novel information on antibody heterogeneity and on its susceptibility to degradation, which can have fundamental implications for the introduction of potential biosimilar candidates in the future.

\section{Challenges}

In industry, charge variant analysis (CVA) is CQAs used for direct comparison of different product batches with innovator to ensure consistent product quality. Recently CVA, both Weak Cation Exchange (WCX) and Strong Cation Exchange (SCX) chromatography is used in a process.

Analytical Technology as a powerful tool to control and optimise upstream and downstream processing in biopharmaceutical industry [5]. One limitation is the necessity to perform elaborate sample preparation procedures and follow-up experiments for initial identification of the peaks and in case of any out-of-specification events. These experimental procedures usually involve peak collection followed by buffer exchange to allow further analysis, which can be time consuming and costly. More importantly, these procedures are a possible source for sample preparation-induced modifications [6].

Conventional IEC uses gradients of low-to-high salt concentration to elute proteins, but when coupling to MS, the high concentrations of salt can be problematic, even when utilizing volatile salts. An additional limitation of this approach is denaturation of the protein, resulting in the generation of complex mass spectra containing highly charged $\mathrm{mAb}$ ions due to the loss of tertiary structure and exposure of charged residues.

Another big challenge for CEX analysis using HPLC is the life of HPLC parts, as traditional method has a salt based approach for analysis of charge variants and it is recommended not to use more salt for HPLC life.

\section{Plan of Action}

- Select Mass compatible volatile buffer system with low buffering capacity.

- Select UPLC column which can be reproducible for high and low $\mathrm{pH}$ gradient.

- Select $\mathrm{pH}$ range for the mobile phase which be used for different mAbs with pl range 7 to 10 .

- Develop chromatographic gradient with high resolution and short run time.

- Optimize TOF Mass Spectrometry parameters for identification of charge species.

- To validate CEX-MS method for transfer in Quality Control Dept.

\section{Initial Study Outcomes}

We are in the middle of developing a powerful tool for online Charge Variant Analysis-Mass Spectrometry using volatile buffers that has been shown to provide a stable $\mathrm{pH}$ gradient, hence facilitating CVA of multiple, commercially available mAb drug products using Triple TOF MS detection [7]. We present a modified buffer system with an expanded application range, enabling the analysis of highly basic mAbs. In addition, by applying the optimal settings for MS data acquisition, we completely mitigated the occurrence of adduction, resulting in a more generic and sensitive method for quick and thorough analysis ofmAb heterogeneity. We demonstrate that data acquisition strongly benefits from higher resolution settings when discrimination of co-eluting near isobaric substances is required.Among them were proteoforms modified by different levels of C-terminal lysine truncation, deamidation, succinimideaspartic acid (Asp) formation, glycation and fragmentation. The ultra-performance liquid chromatography (UPLC)-setup used in this study is fully MS friendly and provides excellent chromatographic selectivity. For most of the mAbs analysed, this results in a variety of fully and partially resolved peaks.

\section{Future Plan of Action}

From the initial study experiment it was observed that volatile mobile phase with $\mathrm{pH}$ gradient formula is working better to resolve acidic and basic peaks. Also, in mass spectrometry the resolution of acidic and basic peaks are detectable till MS-MS. In these studies, glycan profiling indicated the presence of GOF, G1F, GOF/GOF, GOF/G1F and G1F/G1F or GOF/G2F. CVA performed in the course of batch-to-batch comparison revealed the presence of highly abundant basic species, attributed to different C-terminal lysine forms. This analysis also revealed the presence of minor acidic species, which were, however, unidentified. 
Now we are trying to do orthogonal approaches to verify the data observed in CVA-MS analysis. Also, force degradation study need to be performed for acidic and basic variant confirmation in stability study analysis.

\section{References}

1. Kenett RS, Kenett DA (2008) Quality by design applicatiinons in biosimilar pharmaceutical products. Accreditation and Quality Assurance 13: 681-690.

2. Berkowitz SA, Engen JR, Mazzeo JR, et al. (2012) Analytical tools for characterizing biopharmaceuticals and the implications for biosimilars. Nat Rev Drug Discov 11: 527-540.

3. Liu H, Gaza-Bulseco G, Faldu D, et al. (2008) Heterogeneity of monoclonal antibodies. J Pharm Sci 97: 2426-2447.
4. Torkashvand F, Vaziri B (2017) Main quality attributes of monoclonal antibodies and effect of cell culture components. Iran Biomed J 21: 131-141.

5. Patel BA, Pinto NDS, Gospodarek A, et al. (2017) Online ion exchange liquid chromatography as a process analytical technology for monoclonal antibody characterization in continuous bioprocessing. Anal Chem 89: 11357-11365.

6. Griaud F, Denefeld B, Lang M, et al. (2017) Unbiased in-depth characterization of CEX fractions from a stressed monoclonal antibody by mass spectrometry. MAbs 9: 820-830.

7. Füssla F, Trappe A, Cook K, et al. (2020) Comprehensive characterisation of the heterogeneity of adalimumab via charge variant analysis hyphenated on-line to native high resolution Orbitrap mass spectrometry. MAbs 11: 116-128. 